\title{
POLÍMEROS DESAGUADORES PARA DISPOSIÇÃO DE REJEITOS ADENSADOS
}

\author{
R. H. O. BARREDA ${ }^{1}$, G. E. S. VALADÃO \\ Universidad Arturo Prat ${ }^{1}$, Universidade Federal de Minas Gerais ${ }^{2}$ \\ ORCID ID: https://orcid.org/0000-0002-2855-5054 ${ }^{1}$ \\ rolcay@gmail.com ${ }^{1}$
}

Submetido 03/08/2020 - Aceito 20/11/2020

DOI: $10.15628 /$ holos.2020.10884

\begin{abstract}
RESUMO
A prática mais comum para a disposição de rejeitos é a subaquática e vem se tornando um assunto cada vez mais importante e indispensável no gerenciamento de barragens e continuidade operacional das plantas mineradoras devido principalmente aos aspectos como: ambientais, segurança, sociais, econômicos, etc. As técnicas de espessamento e filtragem se apresentam como alternativas para o desaguamento dos rejeitos quando comparada à disposição convencional. O objetivo principal deste trabalho é apresentar uma alternativa diferente às técnicas mencionadas através da utilização de polímeros desaguadores desenvolvidos recentemente. Um rejeito em torno de $10 \%$ sólidos em massa e de granulometria fina com tamanho inferior a $70 \mu \mathrm{m}$ foi avaliada em escala de laboratório mediante
\end{abstract}

ensaios de: espessamento colunar, filtragem (filtro prensa horizontal) e aplicação de polímeros desaguadores. Obtevese pasta com $52 \%$ de sólidos em massa e umidade da torta menor que $25 \%$ para o espessamento e filtragem respectivamente. Mediante o uso do polímero desaguador foi possível a liberação inicial sobre $70 \%$ da água contida no rejeito e obter resultados similares às outras técnicas com o tempo de disposição. Os resultados apresentaram que é possível o uso desta alternativa tecnológica e permitiu estabelecer um comparativo das vantagens e desvantagens entre as outras técnicas mencionadas.

PALAVRAS-CHAVE: Desaguamento, Polímeros, Disposição, Barragem.

\section{DEWATERING POLYMERS FOR THICKENED TAILINGS DISPOSAL}

\begin{abstract}
The most common practice for the disposal of tailings is the subaquatic and has become a subject increasingly important and indispensable in the management of dams and operational continuity of mining plants due mainly aspects as: environmental, safety, social, economic, etc. The thickening and filtration techniques are presented as alternatives for the dewatering of tailings. The main objective of this research is to present an alternative different of the techniques mentioned through the use of dewatering polymers development recentily. A tailings around $10 \%$ solids in mass and fine granulometry with size less $70 \mu \mathrm{m}$ were evaluated in
\end{abstract}

laboratory scale through tests: column thickener, filtration (horizontal press filter) and aplication of dewatering polymers. Was obtained paste with $52 \%$ solids in mass and cake moisture less of $25 \%$ for thickening and filtering respectively. Through the use of dewatering polymers was possible initially release over $70 \%$ of the water contained in the tailings and obtain results similar that the other techniques with the disposal time. The results showed that is possible the application of this technological alternative and allowed establish a comparison of the advantages and disadvantages among the others techniques mentioned.

KEYWORDS: Dewatering, Polymers, Disposal, Dam. 


\section{INTRODUÇÃO}

As atividades de mineração vêm gerando elevadas quantidades de rejeitos, sendo em muitos casos não reaproveitáveis como subprodutos na cadeia produtiva, transformando-se assim em passivos ambientais. A maior parte da disposição de rejeitos da mineração mundial se faz por barragens de rejeitos, cuja função principal é a contenção dos mesmos, tendo por objetivo secundário o armazenamento de água para o reuso na mina e/ou no beneficiamento (IBRAM, 2016). A disposição de rejeitos nas barragens pode apresentar diversos riscos operacionais e ambientais trazendo sérias consequências para a população e para o próprio empreendimento mineral. A disposição na forma de pasta é uma alternativa que apresenta diversas vantagens quando se compara com a disposição convencional, por exemplo, se tem: maior reaproveitamento das áreas de disposição de rejeitos, aumento da recirculação de águas de processo, maior segurança e flexibilidade operacional entre outros fatores (Olcay et al., 2020).

As técnicas de espessamento e filtragem vêm se apresentando há um bom tempo como alternativas para o desaguamento de rejeitos, mas muitas vezes opta-se por manter a disposição convencional pelos menores custos associados. Espessadores de pasta como HiDensity e DeepBed foram desenvolvidos para produzir elevadas tensões de escoamento na descarga do equipamento. Conforme Summerhaysa e Gaspar (2016), a tensão de escoamento é definida como o mínimo esforço que deve ser aplicado a um material de forma a permitir o seu fluxo. Vários estudos fazem uma relação da tensão de escoamento com a concentração de sólidos dos rejeitos (Boger, 2000, 2009, 2013; Sofra \& Boger, 2002; Gawu \& Fourie; 2004; Olcay et al., 2019).

A tensão de escoamento dos rejeitos aumenta exponencialmente com o aumento da concentração de sólidos, portanto, uma pequena alteração na concentração pode resultar em uma grande variação da tensão de escoamento (Gao \& Fourie, 2015), consequentemente, o controle da consistência do material nas operações de disposição de resíduos é crítica (Boger, 1998; Clayton et al., 2003). A Figura 1 apresenta em caráter qualitativo, a tensão de escoamento em função do adensamento de um material em termos de porcentagem de sólidos em massa (Olcay et al., 2020). Diversos estudos relacionam a tensão de escoamento com a estabilidade coloidal e características superficiais das partículas (Dzuy et al., 1983; Coussot, 2005; Baker, 2010).

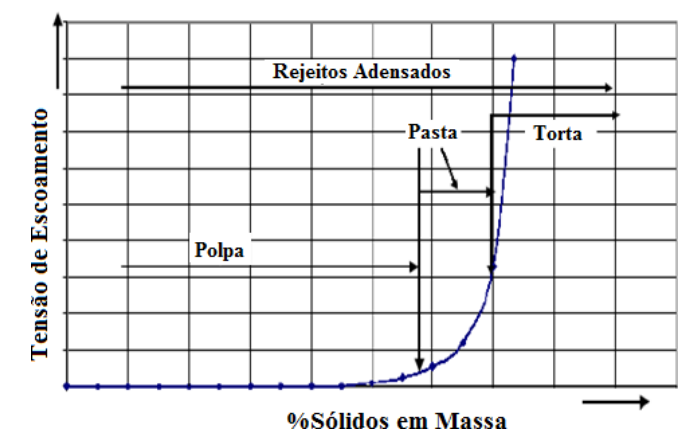

Figura 1: Tensão de escoamento em função da porcentagem de sólidos em massa (Olcay et al, 2020). 
A filtragem é uma técnica comum de separação sólido-líquido de muitas indústrias e pode ser definida como uma operação unitária de separação dos sólidos contidos em uma suspensão aquosa mediante a passagem da polpa através de um meio filtrante, que retém as partículas sólidas e permite a passagem do líquido (usualmente a água). 0 líquido que atravessa o meio filtrante é denominado filtrado e os sólidos retidos constituem a torta. Os filtros posuem uma variedade de geometrias e mecanismos (Valadão \& Araujo, 2012; Guimarães et al., 2012; Stickland et al., 2018).

Segundo Tão et al. (2008), na indústria de minerais fosfáticos a disposição de lamas apresenta dificuldades devido a sua natureza coloidal que a tornam muito estáveis em suspensões aquosas com uma sedimentação extremamente lenta. A consolidação de lamas fosfáticas em um estado adequado para recuperação e reutilização de terras pode levar uma década ou mais. Esforços para resolver os problemas associados à disposição e recuperação de lamas fosfáticas incluem testes mecânicos, elétricos, biológicos e processos químicos. Esses esforços consideram a adição de coagulantes e floculantes, desaguamento mecânico, o uso de enzimas e bactérias como também vários outros métodos de espessamento e estabilização (Les et al., 2011).

De acordo com Qi et al. (2011), a lama é um sistema coloidal no qual pequenas partículas formam uma suspensão estável na água e a separação entre sólido e líquido é muito difícil. A adição de reagentes químicos tais como floculantes e coagulantes é muitas vezes necessário para auxiliar na agregação das partículas. A floculação consiste em um fenômeno físico-químico que visa alterar as propriedades estáveis de um coloide, tornando-o instável a ponto de formar flocos, cujos diâmetros são maiores do que as partículas isoladas. Essa instabilidade é provocada pela inserção de um polímero no seio do fluido. Esse polímero possui sítios ativos em sua superfície que, dependendo da carga dos sólidos envolvidos no processo, irá atraí-los formando agregados moleculares (Sharma et al., 2006; Baltar, 2010).

Em conformidade com Wei et al. (2018), a tecnologia de disposição de lamas se encontra enfrentando simultaneamente desafios e oportunidades que ainda podem ser melhorados. 0 desaguamento de lamas é um processo essencial na disposição de rejeitos, sendo muito importante para a redução efetiva dos custos finais do beneficiamento mineral. A eficiente separação sólido-líquido das lamas é um dos passos chaves para reduzir os custos com o tratamento, transporte e disposição final (Mowla et al., 2013).

O presente trabalho tem como objetivo apresentar um comparativo de diferentes mecanismos de desaguamento de rejeitos fosfáticos utilizando as técnicas de espessamento, filtragem e utilização do polímero desaguador, visando obter diferentes apreciações das alternativas de desaguamento para disposição de rejeitos no gerenciamento de barragens. 


\section{METODOLOGIA}

\subsection{Amostra}

A amostra utilizada representa um rejeito total proveniente de uma usina de beneficiamento de minério fosfático localizada no Estado de Minas Gerais. A Tabela 1 apresenta as diferentes técnicas utilizadas para a caracterização da amostra.

Tabela 1: Técnicas e equipamentos na caracterização da amostra.

\begin{tabular}{ccc}
\hline Propriedade a determinar & Técnica utilizada & Equipamento Utilizado \\
\hline Granulometria & Difração Laser & Analisador Partículas (Cilas) \\
\hline Peso específico & Picnometría & Picnômetro Simples \\
\hline Composição química & Fluorescência raios X & Espectrômetro raios X dispersivo \\
\hline Consistência & Ângulo Empilhamento & Cilindro PVC \\
\hline
\end{tabular}

\subsection{Ensaios no Espessador Colunar Contínuo}

Os ensaios de espessamento foram realizados em um espessador colunar em escala de laboratório com um volume total de 10L, mediante alimentação contínua com aproximadamente $10 \%$ de sólidos em massa e recirculação da polpa. A Figura 2 apresenta uma vista geral para o desaguamento do rejeito total com uso de polímero convencional e espessador colunar.

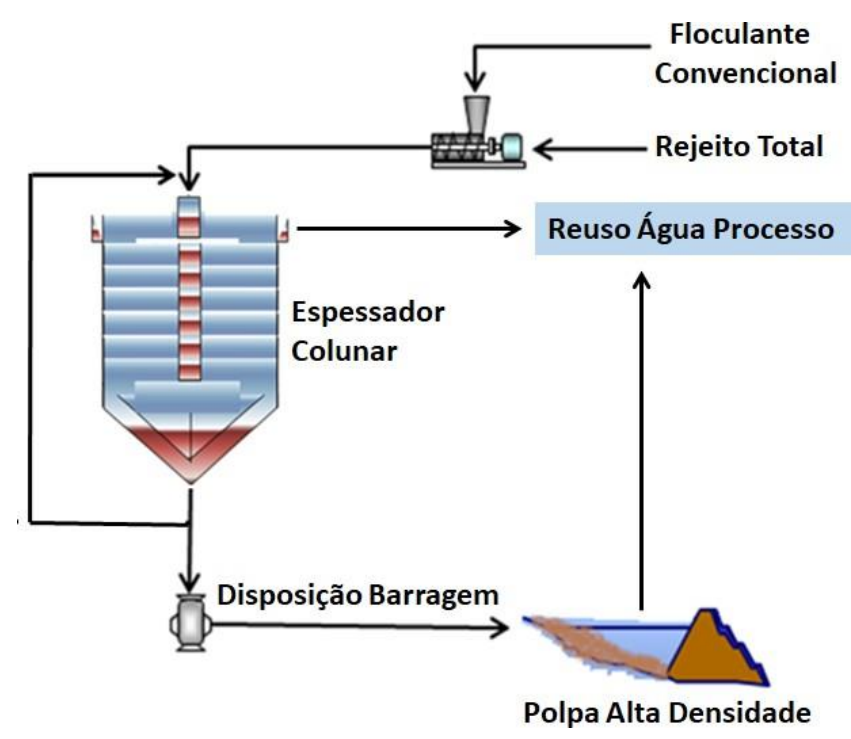

Figura 2: Espessamento com polímero convencional do rejeito total para disposição superficial 


\subsection{Ensaios de Filtragem Horizontal Descontínua}

Os ensaios de filtragem horizontal descontínua foram realizados após espessamento convencional da amostra ( $20 \%$ sólidos em massa) mediante uso de filtro prensa horizontal tipo recesso em escala de laboratório, os ensaios foram realizados para diferentes condições operacionais em pressões da alimentação da polpa, tempos de prensagem e secagem, em procura dos melhores parâmetros operacionais e visando simular o circuito de desaguamento do rejeito total conforme apresentado na Figura 3.

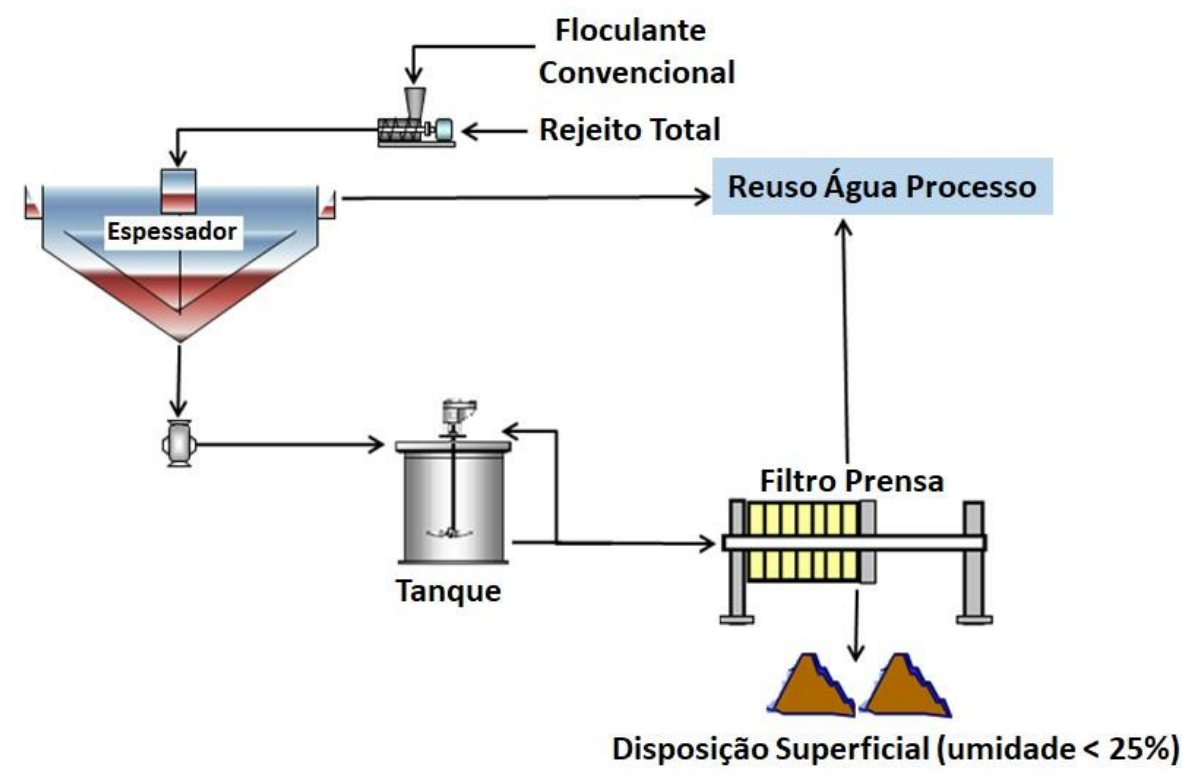

Figura 3: Espessamento e filtragem do rejeito total para disposição superficial a seco.

\subsection{Ensaios Polímero Desaguamento}

Foram utilizadas para cada ensaio amostras com aproximadamente $10 \%$ de sólidos em massa. Os polímeros desaguadores avaliados são poliacrilamidas aniônicas e foram preparados na concentração de $0.25 \% \mathrm{~m} / \mathrm{v}$ mediante o uso de agitador magnético. Uma vez preparados os polímeros desaguadores foram adicionados na dosagem requerida no béquer que contém a amostra. A mistura da amostra com o polímero desaguador avaliado foi realizada mediante o uso de dois béqueres de $0.5 \mathrm{~L}$, onde foi transferida a amostra com o polímero desaguador contida em um béquer para outro vazio e vice-versa por três vezes, logo foi retirado o sobrenadante do béquer e preenchido o recipiente de PVC (altura $=$ diâmetro $=50 \mathrm{~mm}$ ) para determinar o ângulo de empilhamento de cada amostra ensaiada. A seleção do polímero desaguador foi baseada através dos ângulos de empilhamento obtidos a partir dos ensaios de consistência utilizado para suspensões minerais (Clayton et al., 2003; Boger 2009; Mizani e Simms, 2016; Trampus e França, 2019) conforme apresentado na Figura 4. 


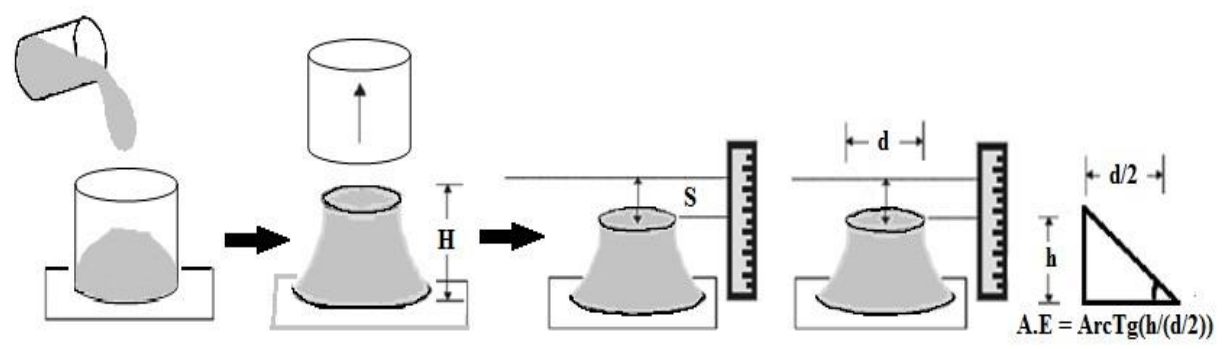

Figura 4. Procedimento para determinação do ângulo de empilhamento.

A Figura 5 apresenta de forma geral uma vista da usina de beneficiamento com a aplicação do polímero desaguador no rejeito total para disposição na barragem, cabe destacar que geralmente na operação a mistura do polímero desaguador com o rejeito é realizada na linha de descarga do rejeito.

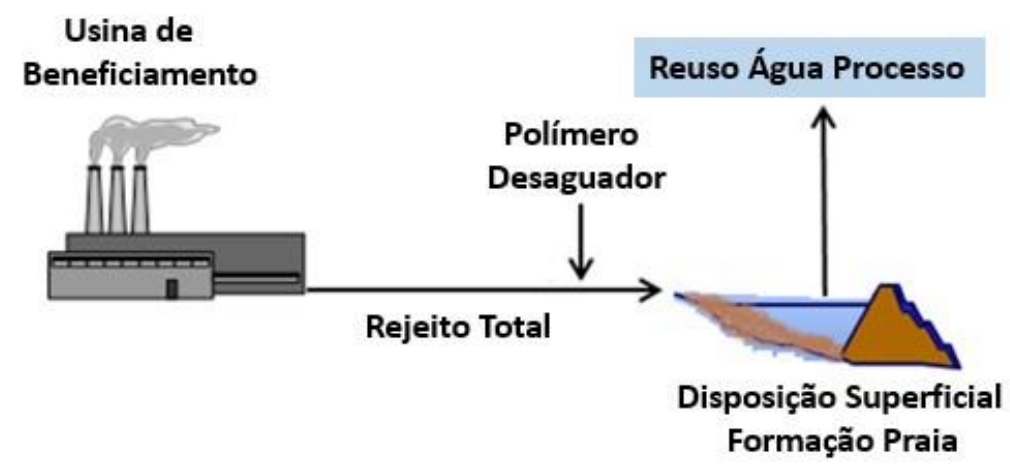

Figura 5: Disposição do rejeito total com aplicação de polímero desaguador.

\subsection{Medição da Tensão de Escoamento}

Para as medições da tensão de escoamento das amostras foi utilizado o modelo de Bingham mediante o método de Vane que consiste basicamente em utilizar uma haste de quatro pás que é imersa em um recipiente contendo a amostra até uma determinada profundidade. A força de giro foi aumentada até alcançar o máximo valor e diminuída gradualmente até deter a leitura. $\mathrm{O}$ valor máximo foi utilizado como a tensão de escoamento. A Figura 6 apresenta o reômetro utilizado.

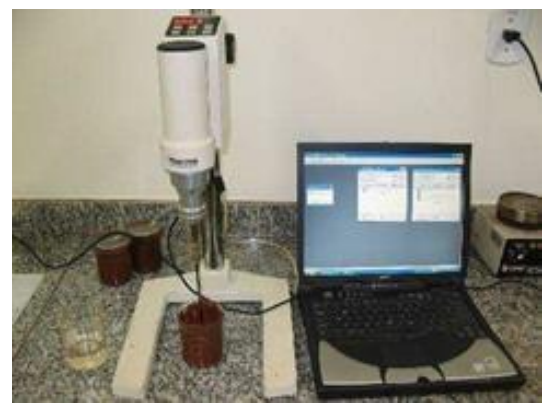

Figura 6: Reômetro Haake (VT-550) para a medição da tensão de escoamento. 


\section{RESULTADOS E DISCUSSÃO}

\subsection{Amostra}

\subsubsection{Granulometria}

A Figura 7 se apresenta a distribuição granulométrica da amostra ensaiada.

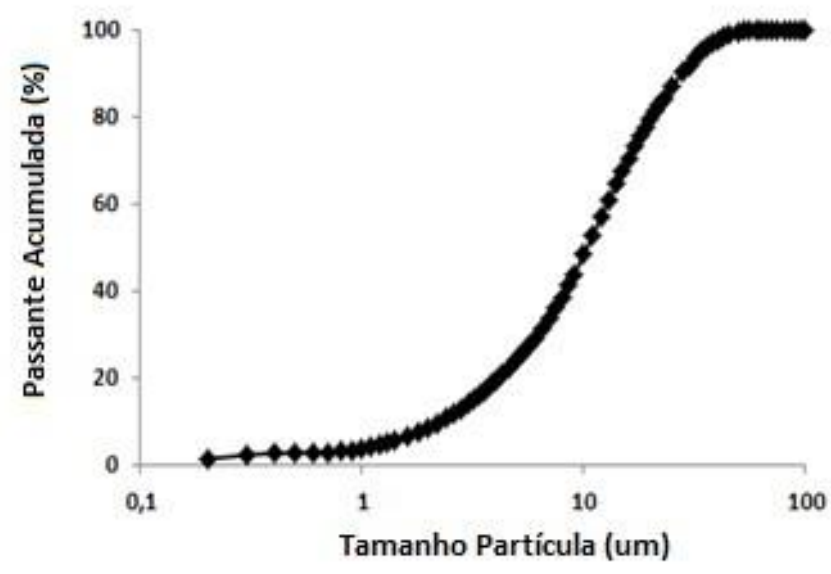

Figura 7: Distribuição tamanho de partículas da amostra.

Como pode ser observado na Figura 7 a amostra é muito fina, sendo na sua totalidade de tamanho inferior a $70 \mu \mathrm{m}$. O tamanho médio da amostra $\left(\mathrm{d}_{50}\right)$ em torno de $10.5 \mu \mathrm{m}$.

\subsubsection{Peso Específico}

O peso específico médio da amostra, obtido por meio de picnometría simples, foi igual a $2,84 \mathrm{~g} / \mathrm{cm}^{3}$ conforme apresentado na Tabela 2 .

Tabela 2: Medições de peso específico da amostra.

\begin{tabular}{lcccc}
\hline Medição & $\mathbf{1}^{\circ}$ & $\mathbf{2}^{\circ}$ & $\mathbf{3}^{\circ}$ & Média \\
\hline Peso Específico $\left(\mathrm{g} / \mathrm{cm}^{3}\right)$ & 2.86 & 2.82 & 2.85 & $\mathbf{2 . 8 4}$ \\
\hline
\end{tabular}

\subsubsection{Composição Química}

A composição química dos principais componentes presentes na amostra foi obtida por meio de fluorescência de raios X. A Tabela 3 apresenta os resultados obtidos.

Tabela 3: Composição química dos principais componentes na amostra.

\begin{tabular}{ccccc}
\hline Composto & $\%$ & Composto & $\%$ \\
\cline { 1 - 2 } $\mathrm{SiO}_{2}$ & 62.12 & & $\mathrm{Fe}_{2} \mathrm{O}_{3}$ & 5.89 \\
\cline { 1 - 2 } \cline { 5 - 6 } $\mathrm{Al}_{2} \mathrm{O}_{3}$ & 13,42 & & $\mathrm{P}_{2} \mathrm{O}_{5}$ & 2.97 \\
\cline { 1 - 2 } $\mathrm{CaO}$ & 10.44 & & $\mathrm{MgO}$ & 1.10 \\
\hline
\end{tabular}

Conforme pode ser observado na Tabela 3 foi evidenciada a predominância de $\mathrm{SiO}_{2}, \mathrm{Al}_{2} \mathrm{O}_{3}$, $\mathrm{CaO}$ e $\mathrm{Fe}_{2} \mathrm{O}_{3}$ com a presença em menores quantidades de $\mathrm{P}_{2} \mathrm{O}_{5}$ e $\mathrm{MgO}$. 


\subsection{Ensaios espessamento colunar contínuo}

A porcentagem de sólidos na alimentação foi em torno de $10 \%$ sólidos em massa, as concentrações de sólidos em massa obtidas na descarga do espessador colunar foram obtidas para diferentes tempos de operação contínua, chegando até 120 minutos com dosagem de $90 \mathrm{~g} / \mathrm{t}$ de polímero convencional preparado a uma concentração de $0.1 \% \mathrm{~m} / \mathrm{v}$. A Figura 8 apresenta as porcentagens de sólidos em massa obtidas em função do tempo de operação contínua.

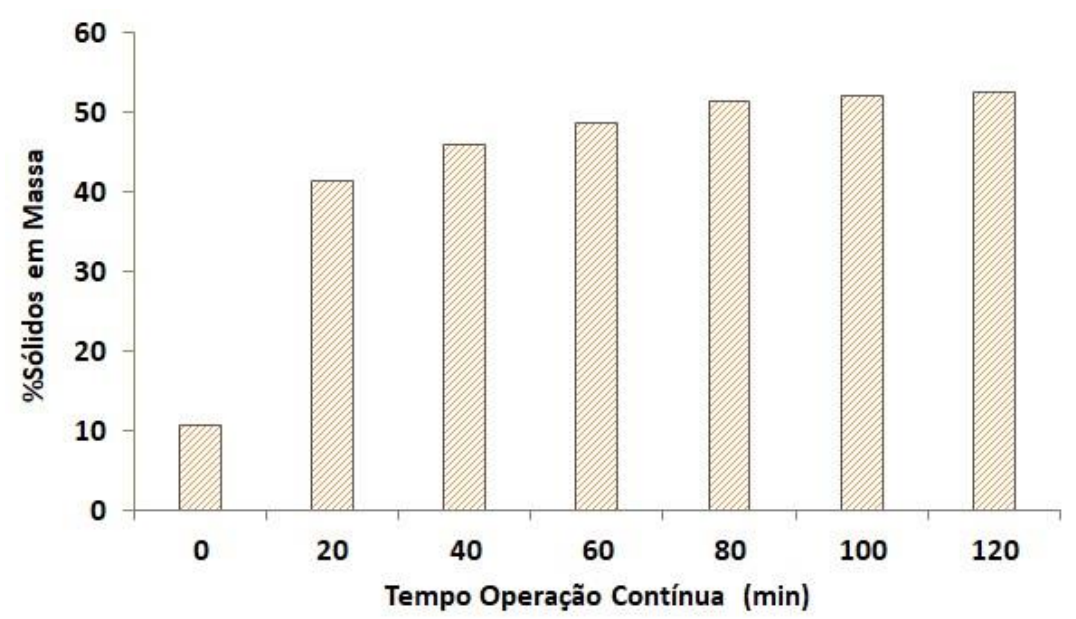

Figura 8: \%Sólidos em massa na descarga do espesador colunar em função do tempo operação contínua.

Segundo observado na Figura 8 as maiores concentrações de sólidos em massa obtidas na descarga do equipamento foram desde 80 minutos de operação contínua, com aproximadamente um $52 \%$ de sólidos em massa, as medições da tensão de escoamento indicaram valores em torno de 210Pa. Desta forma, o rejeito adensado através desta técnica pode ser classificado como tendo uma reologia de pasta mineral (Boger et al., 2005). Cabe destacar que os valores obtidos para a turbidez de overflow, nestes ensaios, apresentaram uma turbidez menor que 100NTU permitindo sua reutilização no processo de beneficiamento.

\subsection{Ensaios de Filtragem Descontínua}

A Figura 9 apresenta as taxas unitárias de filtragem obtidas no filtro prensa horizontal com as melhores condições operacionais, sem e com uso de cal hidratada como auxiliar de filtragem para obtenção de tortas com valores menores que $25 \%$ de umidade total.

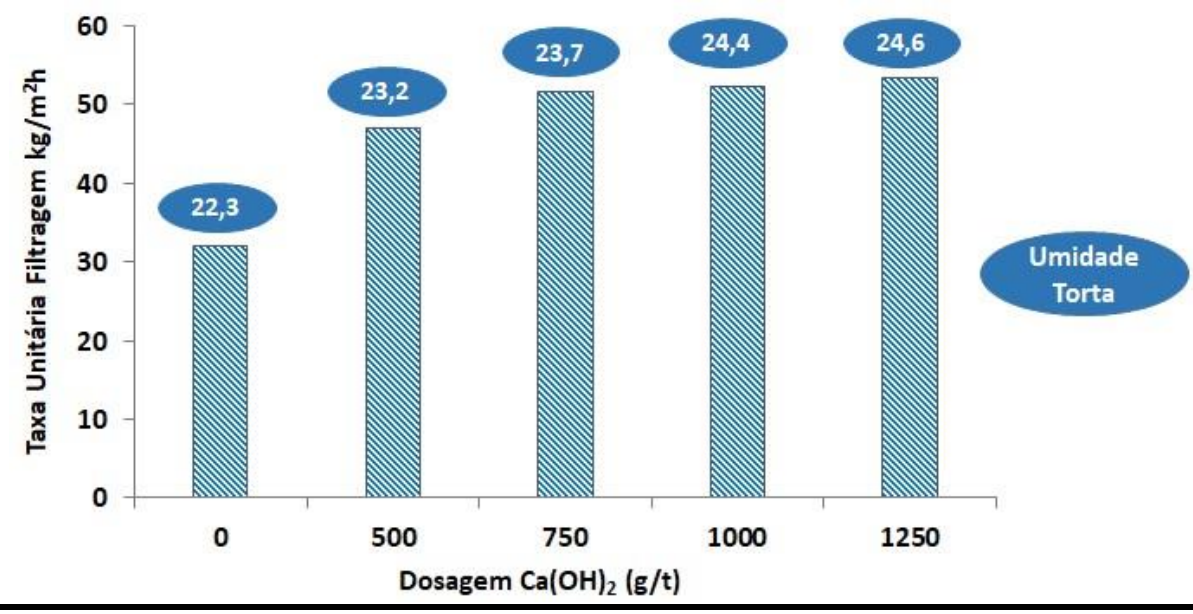


Figura 9: Taxa unitária de filtragem em função da dosagem de cal hidratada.

Observa-se na Figura 9 que para a obtenção de uma torta com um máximo de $25 \%$ de umidade sem uso de cal hidratada a taxa de filtragem foi em torno de $30 \mathrm{~kg} / \mathrm{m}^{2} \mathrm{~h}$. As melhores taxas unitárias de filtragem foram da ordem de $50 \mathrm{~kg} / \mathrm{m}^{2} \mathrm{~h}$, mas foi requerida a aplicação do auxiliar de filtragem com dosagens desde $750 \mathrm{~g} / \mathrm{t}$. Verifica-se que nas melhores condições operacionais as tortas tiveram uma umidade menor a $25 \%$ e filtrados com valores de turbidez menores a 100 NTU, fato que facilita sua reutilização no processo de beneficiamento mineral. Os valores das taxas unitárias de filtragem obtidas foram considerados como baixas frente ao mínimo esperado $200 \mathrm{~kg} / \mathrm{m}^{2} \mathrm{~h}$, possivelmente devido a uma maior dificuldade na filtragem pela elevada presença de partículas finas na amostra. A Figura 10 apresenta uma torta obtida nos ensaios de filtragem com a polpa bombeada para as placas membranas com: $20 \%$ sólidos em massa, temperatura polpa alimentada $25^{\circ} \mathrm{C}$, pressão da câmara 7 bar e uso de ar comprimido para secagem da torta.
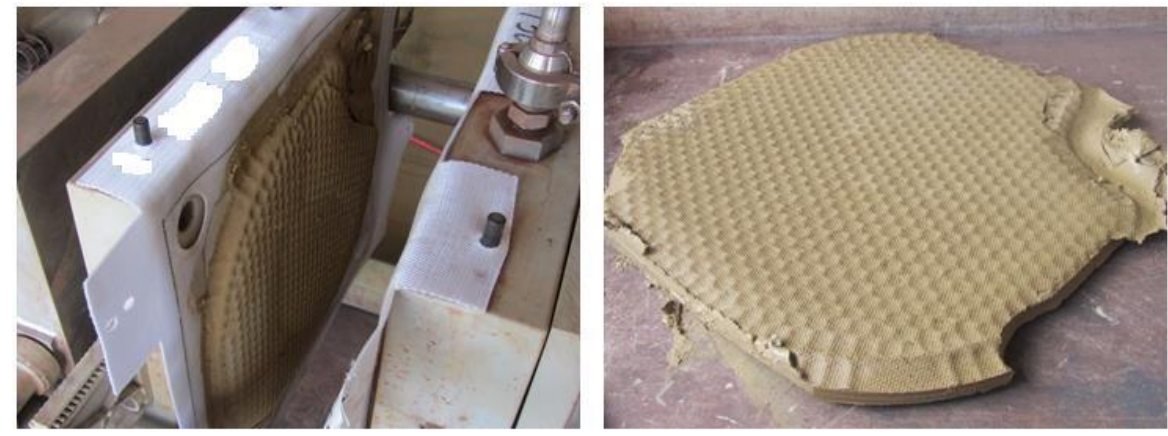

Figura 10: Torta obtida nos ensaios de filtragem.

\subsection{Ensaios Polímero Desaguador}

A seleção do polímero desaguador e da dosagem requerida foi baseada nos ângulos de empilhamento obtidos através dos ensaios de abatimento da amostra.

A Figura 11 apresenta uma visão geral da amostra sem e com aplicação de diferentes dosagens do polímero desaguador selecionado.
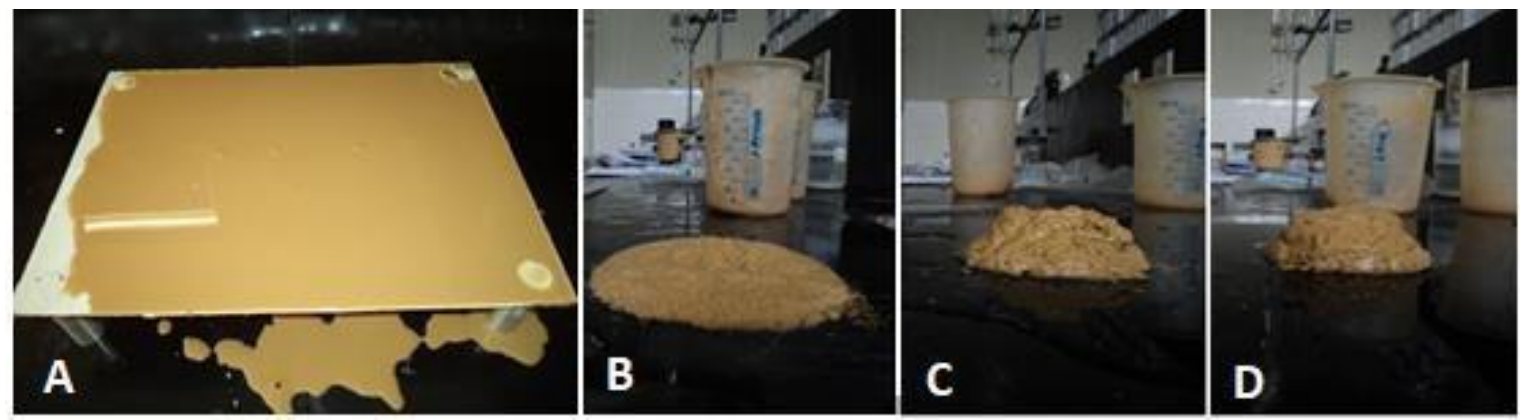

Figura 11: Amostra sem e com aplicação de diferentes dosagens do polímero desaguador: A) dosagem: $0 \mathrm{~g} / \mathrm{t}$; B) dosagem: $100 \mathrm{~g} / \mathrm{t}$; C) dosagem: $200 \mathrm{~g} / \mathrm{t}$; D) $250 \mathrm{~g} / \mathrm{t}$.

A Figura 12 apresenta o desempenho do polímero desaguador selecionado através do ângulo de empilhamento em função de diferentes dosagens aplicadas na amostra. 


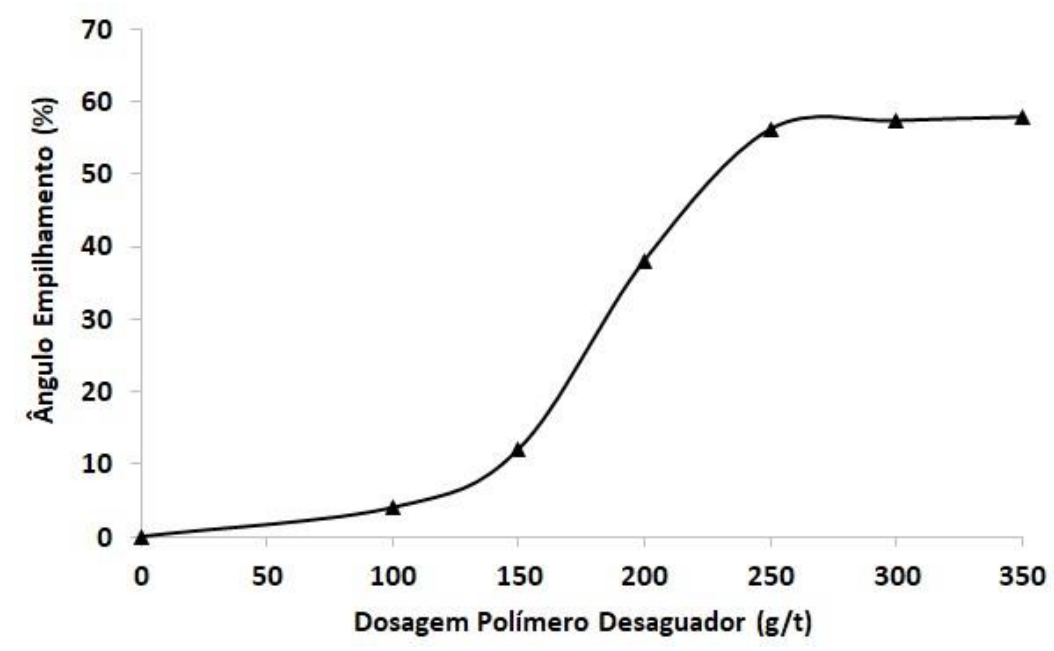

Figura 12: Ângulo empilhamento em função da dosagem do polímero desaguador.

Os maiores ângulos de empilhamento obtidos para a amostra foram com dosagens a partir de $200 \mathrm{~g} / \mathrm{t}$, com ângulos de empilhamento entre $40 \%$ e $60 \%$. Cabe destacar que para se obter sobrenadantes com turbidez abaixo de 100NTU foi necessária a adição de cal hidratada em torno de $1500 \mathrm{~g} / \mathrm{t}$ na amostra antes da mistura com o polímero desaguador.

A Figura 13 apresenta a evolução do desaguamento da amostra no laboratório com uso de polímero desaguador em função do tempo (dias), sob condições de temperatura e pressão ambiente. Observa-se que a amostra tinha uma concentração inicial de sólidos em massa igual a $10.6 \%$.

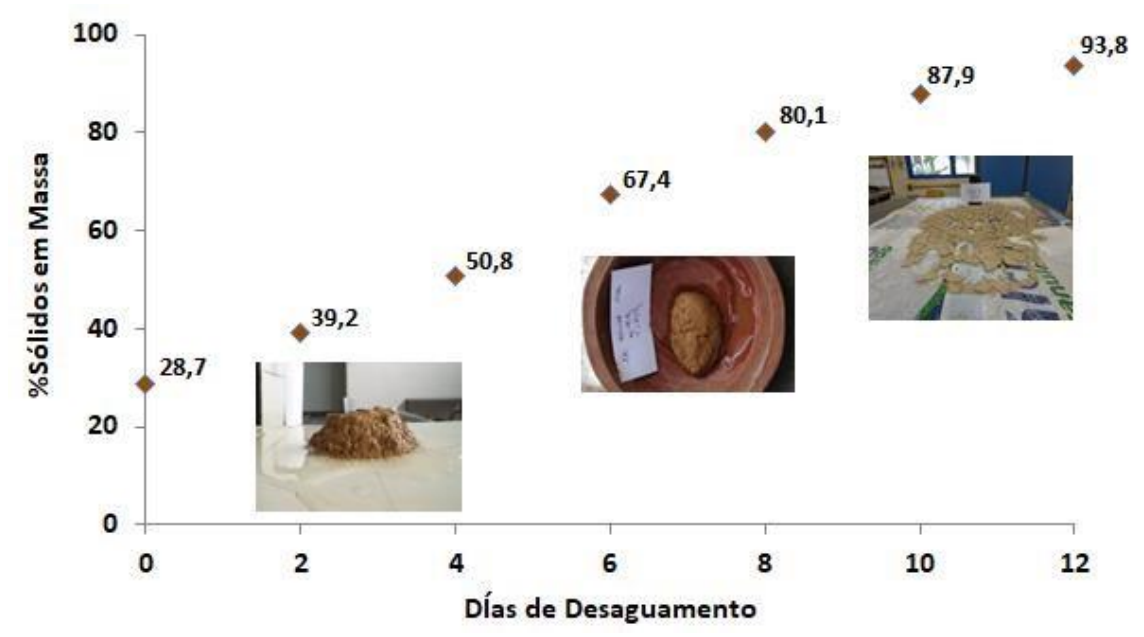

Figura 13: \%Sólidos em massa em função do tempo de desaguamento.

Pode ser observado na Figura 13 que a amostra teve inicialmente um aumento desde $10.6 \%$ a $28.7 \%$ de sólidos em massa, é dizer, liberou mais de $70 \%$ da água contida na polpa após 300 segundos da mistura com do polímero desaguador. Depois do segundo dia, a amostra apresentou características de polpa de alta densidade, após o quarto dia apresentou características reológicas de pasta e a partir do oitavo dia a amostra teve características de torta. 


\subsection{Tensão de Escoamento}

As medições da tensão de escoamento da amostra para diferentes concentrações de sólidos foram obtidas através do modelo de Bingham que são apresentadas na Figura 14.

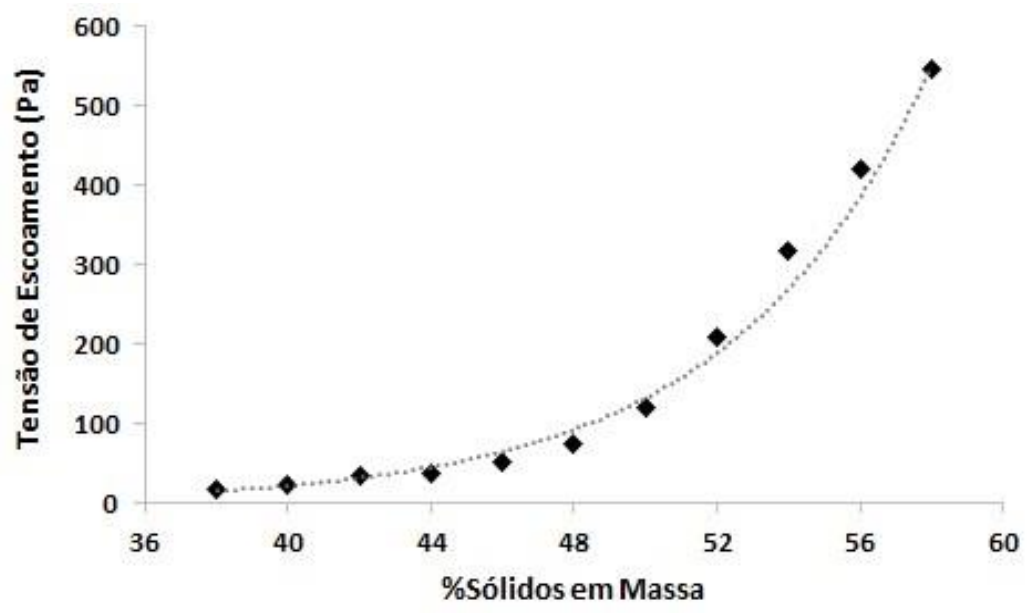

Figura 14: Tensão de escoamento em função da concentração de sólidos em massa.

Observa-se na Figura 14 que a partir de $46 \%$ de sólidos em massa se tem um notável incremento na tensão de escoamento da amostra, para 52\% de sólidos em massa se forma pasta mineral com um valor da tensão de escoamento em torno a $210 \mathrm{~Pa}$. O modelo de Bingham apresentou uma boa tendência exponencial da tensão de escoamento (Te) e a concentração de sólidos em massa, obtendo-se assim a seguinte Equação (1), onde, $x$ é a porcentagem de sólidos em massa da amostra.

$$
\mathrm{Te}=0,0182 \exp ^{0,1779 x}
$$

\section{CONCLUSÕES}

A amostra apresentou uma granulometria muito fina dificultando o desaguamento desta mediante filtragem. Os resultados da tensão de escoamento indicaram que a amostra se apresenta como polpa de alta densidade, pasta mineral e torta para valores sobre: $40 \%, 50 \%$ e $75 \%$ sólidos em massa respectivamente. Mediante o uso do espessador colunar foram obtidas pastas com $52 \%$ de sólidos em massa e com o filtro prensa horizontal se obtiveram tortas com umidade menor a $25 \%$ nas melhores condições operacionais.

A utilização do polímero desaguador apresentou uma boa capacidade de aglomeração de partículas finas e rápida liberação da água presente na polpa, com quantidades de água liberada sobre $70 \%$, que podem ser reutilizadas no processo de beneficiamento ao igual das outras técnicas, apresentando de esta forma ser uma alternativa a ser considerada na disposição de rejeitos adensados nas barragens.

Os polímeros desaguadores avaliados na amostra em escala de laboratório apresentaram que podem ser atingidas as mesmas características reológicas obtidas mediante as técnicas de espessamento e filtragem, mas é necessário um maior tempo de disposição para o desaguamento 
natural da polpa, em conjunto com boas condições climáticas e sistemas de drenagem favoráveis, entre outros fatores.

A filtragem apresentou um maior desaguamento da amostra, mas teve uma baixa taxa unitária de filtragem, seguida do espessamento colunar e polímero desaguador. Cabe mencionar que os custos associados ao uso de polímeros desaguadores referente a equipamentos são menores em comparação às outras técnicas e pode ter aplicações industriais no curto prazo pela facilidade de aplicação.

\section{REFERÊNCIAS BIBLIOGRÁFICAS}

Baltar, C.A.M. (2010). Processos de Agregação. In Tratamento de minérios, Editores LUZ, A.B., SAMPAIO, J.A., FRANÇA, S.C.A. 5ạ Ed; CETEM/MCT, Rio de Janeiro, p. 559-587.

Baker, J.M. (2010). Nonlinear rheology of colloidal dispersions, Journal of Physics: Condensed matter 22(36).

Boger, D.V. (1998). Environmental rheology and the mining industry. Proceedings of the Sixth International Symposium on Mining with Backfill. The Australasian Institute of Mining and Metallurgy Publication Series, Brisbane, Australia, 15-17.

Boger, D.V. (2000). Rheology and the minerals industry, Minerals Processing Extractive Metallurgy. Rev. 20, 1-25.

Boger. D., Scales, P., Sofra, F. (2005). Paste and Thickened Tailings - A Guide: Second Edition, Editors: Jewell, R., Fourie, A. Rheological concepts Chapter 3.

Boger. D.V. (2009). "Rheology and the resource industries". Chemical Engineering Science.

Boger, D.V. (2013). Rheology of slurries and environmental impacts in the mining industry, Annual review of chemical and biomolecular engineering, 4, 239-257.

Clayton, S., Grice, T. G., Boger, D. V. (2003). Analysis of the Slump Test for on-site Yield Stress Measurement of Mineral Suspensions. International Journal of Mineral Processing, Melbourne-Australia, 70, 3-21.

Coussot, P. (2005). Rheometry of Pastes, Suspensions, and Granular Materials: Applications in Industry and Environment, John Wiley \& Sons.

Dzuy, N.Q., Boger, D.V. (1983). Yield Stress Measurement for Concentrated Suspensions, Journal of Rheology, 27, 321-349.

Gao, J., Fourie, A. (2015). Using the flume test for yield stress measurement of thickened tailings, Minerals Engineering, 81, 116-127, 2015.

Gawu, S.K., Fourie, A. (2004). Assessment of the modified slump test as a measure of the yield stress of high-density thickened tailings. Can. Geotech. J, 41, 39-47. 
Gauto, M. A., Rosa, G. R. (2011). Processos e operações unitárias da indústria química. Rio de Janeiro: Ciência Moderna.

Guimarães, N.G., Valadão, G.E., Peres, A.E.C. (2012) Filtragem de rejeitos de minério de ferro visando à sua disposição em pilhas REM: R. Esc. Minas, Ouro Preto, 65(4), 543-548.

INSTITUTO BRASILEIRO DE MINERAÇÃO: IBRAM. (2016). Gestão e Manejo de Rejeitos da Mineração. (1a ed). Brasília.

Les, G.B., Leon, C.S., John, E. (2011). Development and Pilot-Scale Demonstration of Deep Cone Paste Thickening Process for Phosphatic Clay Disposal: Phase II. Institute of Phosphate Research.

Mizani, S., Simms, P. (2016). Method-dependent variation of yield stress in a thickened gold tailings explained using a structure based viscosity model. Minerals Engineering, 98, 40-48.

Mowla D., Tran H., Allen, D. (2013). A review of the properties of biosludge and its relevance to enhanced dewatering processes. Biomass Bioenergy, 58, 365-378.

Olcay, R.H, Hernández, C.A., Valadão, G.E.S, Chavez, A.P. (2020). Production of minerals paste from phosphates sludges, Ingeniare, Revista Chilena de Ingeniería, Universidad de Tarapacá, Chile, $8(2)$.

Olcay, R. H, Hernández, C. A, Valadão, G. E. S. (2019). Slurry dewatering for the disposal in tailings dams. VI International Seminar on Tailings Management. Innovations in conventional, thickened and paste tailings, 6, 98-105.

Qi Y., Thapa K., Hoadley A. (2011). Application of filtration aids for improving sludge dewatering properties - A review, Chemical Engineering Journal, Elsevier, 171, 373-384.

Stickland, A.D., Skinner, S.J., Cavalida, R.G., Scales, P.J. (2018). Optimisation of filter design and operation for wastewater treatment Sludge, Separation and Purification Technology, Elsevier, 198, 31-37.

Summerhaysa, R., Gaspar, A., (2016). Symphos 2015, $3^{\text {rd }}$ International Symposium on Innovation and Technology in the Phosphate Industry. Thickening, Filtration and Clarification in the Phosphoric Acid Industry, Elsevier ScienceDirect, Procedia Engineering, 138, 164-173.

Sofra, F., Boger, D.V. (2002). Environmental rheology for waste minimisation in the minerals industry. Chemical Engineering Journal, 86, 319-330.

Sharma, B.R., Dhuldhoya, N.C., Merchant, U.C. (2016). Flocculants Ecofriendly Approach. Journal of Polymers and the Environment, 14, 195- 202.

Trampus B.C., França, S.C.A. (2019). Rheology applied to the environmental management of mineral tailings: focus on safer disposal and water reuse. International Engineering Journal REM, 72(2). 
Tão, D., Parekh, B.K., Honaker, R. (2008). Development and Pilot-Scale Demonstration of Deep Cone Paste Thickening Process for Phosphatic Clay Disposal: Phase I. Institute of Phosphate Research, 1-17.

Valadão, G.E.S., Araujo, A.C. (2012). Introdução de tratamento de minérios. (1a Ed.). Editora: UFMG, Belo Horizonte.

Wei H., Gao B., Ren J., Li A., Yang, H. (2018) Coagulation/flocculation in dewatering of sludge: A review, Water Research Elsevier, 143, 608-631.

\section{COMO CITAR ESTE ARTIGO:}

Barreda, R. H. O., Valadão, G. E. S. (2020). Polímeros desaguadores para disposição de rejeitos adensados. Holos. 36(8), 1-14.

\section{SOBRE OS AUTORES}

\section{R. H. O. BARREDA}

Acadêmico-Investigador em Metalurgia Extrativa - Tecnologia Mineral.

E-mail: rolcay@gmail.com

ORCID ID: https://orcid.org/0000-0002-2855-5054

\section{G. E. S. VALADÃO}

Professor do Departamento de Engenharia de Minas da Universidade Federal de Minas Gerais.

E-mail: gvaladao@demin.ufmg.br

ORCID ID: https://orcid.org/0000-0002-1563-8897

Editor(a) Responsável: Elenice Schons Silva

Pareceristas Ad Hoc: Otávia Rodrigues e Daniela Horta

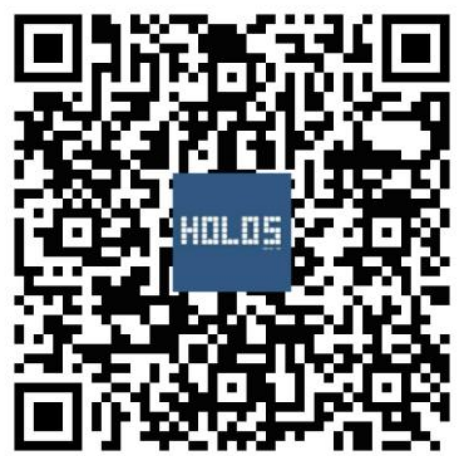

